Short communication

\title{
Ipsilateral hyperschematia without spatial neglect after right frontal lesion ${ }^{\text {零 }}$
}

\author{
Arnaud Saj *, Sabina Catalano Chiuvé, Camille Brugger, Marian van der Meulen, Frédéric Assal \\ Neuropsychology Unit, Neurology Department, University Hospital of Geneva and Faculty of Medicine, Switzerland
}

\section{A R T I C L E I N F O}

Article history:

Received 9 May 2011

Received in revised form 14 June 2011

Accepted 21 June 2011

Available online $\mathrm{xxxx}$

\section{Keywords:}

Hyperschematia

Frontal lesion

Space distortion

\begin{abstract}
A B S T R A C T
The disorder is described as a size distortion involving the side of space. We report the case of a woman with an ipsilateral hyperschematia without neglect after a right frontal lesion. The patient has exhibited a disproportionate enlargement of the right-hand side of objects and added more left-sided petals to the drawn daisy. This pathologic behavior is independent from spatial neglect and from classic frontal perseverations. Our data support the presence of a spatial component to the perception of object size and a specific subcomponent of space distortion.
\end{abstract}

(C) 2011 Elsevier B.V. All rights reserved.

\section{Introduction}

The term 'hyperschematia' was introduced in the neurological literature in 1905 by Bonnier, to describe patients who overestimate the size of the whole body or part of it [1,2]. Unilateral and particularly left-sided hyperschematia now refers to a disproportionate left-sided expansion in drawing tasks, which has been observed in patients with right temporo-parieto-occipital lesions [3,4]. Here, we report the first case of a patient presenting with ipsilateral hyperschematia after a right frontal lesion.

\section{Case report}

A 65-year-old right-handed woman, with 20 years of education, and no history of neurological or psychiatric disorders, was admitted to the stroke unit for a sudden onset of confusion with temporospatial disorientation and nausea, secondary to a right frontal hematoma (Fig. 1A). The lesion includes the dorsolateral prefrontal cortex (BA9, BA46), the frontal eyefield (BA8), the inferior frontal gyrus (BA44, BA45), the orbito-frontal cortex (BA47, BA11), the frontopolar cortex (BA10), the premotor cortex (BA6), and the anterior cingulea including its dorsal (BA32) and ventral regions (BBA24). The patient signed an informed consent form following the principles outlined in the Helsinki Declaration before taking part in the study. On examination, she presented with anosognosia, apathy,

\footnotetext{
Disclosure: The authors report no conflict of interest. All authors have full access to all the data in the study and take responsibility for the integrity of the data and the accuracy of the data analysis. This work was supported by the Swiss National Foundation for AS (\#3200B0-114014).

* Corresponding author at: Dept of Neurology, HUG, Rue Gabrielle-Perret-Gentil 4, CH-1211 GENEVA 14, Switzerland. Tel.: +4122 379 5381; fax: + 41223795402.

E-mail address: arnaud.saj@unige.ch (A. Saj).
}

emotional blunting, rightward deviation of head and trunk, reduced spontaneous utilization of the left limbs (motor neglect), marked psychomotor slowing with deficits in executive functions (letter fluency, TMT part A and B) and working memory (verbal: 5; visuospatial: 3). Importantly, there was no evidence of neglect in peripersonal space in a line cancelation task (7.8\%; cut-off $11 \%)$, in a bell cancelation task (left omission: 3; right omission: 2 ) and in the copying of a complex scene (4/4), and no graphic or motor perseverations were observed.

Next, the patient was asked to draw a flower from memory (Fig. 1B) on a $21 \times 29.7 \mathrm{~cm}$ sheet of paper that was aligned with the midsagittal plane of her body. Using a similar evaluation method to the one used by Rode et al. [3], we measured the number of petals on the left and on the right side, after dividing the flower into two equal halves, by drawing a vertical line passing through the center of its pistil. Petals that fell onto both sides of the vertical line were not considered. The number of petals drawn was 12 on the left side and 35 on the right side. When asked to draw a cube, the patient clearly expanded the right part of it (Fig. 1C), and when drawing an unfolded cube, she only stopped adding additional sides to the right when there was no more paper left. (Fig. 1D).

\section{Discussion}

Bonnier described only briefly [5], his cases of hyperschematia, which occurred in patients with peripheral disorders of the vestibular system. In these cases, the phenomenon was not lateralized or global ("he felt his head becoming huge, immense"). Other published cases all disclosed left-sided (contralateral) hyperschematia, accompanied by left-sided spatial neglect and associated with right posterior lesion $[3,4]$. Moreover, size distortions in perceptual judgments have been described only in patients with spatial neglect after right hemispheric lesions [5]. As suggested by Rode et al. [3,4], hyperschematia can be 

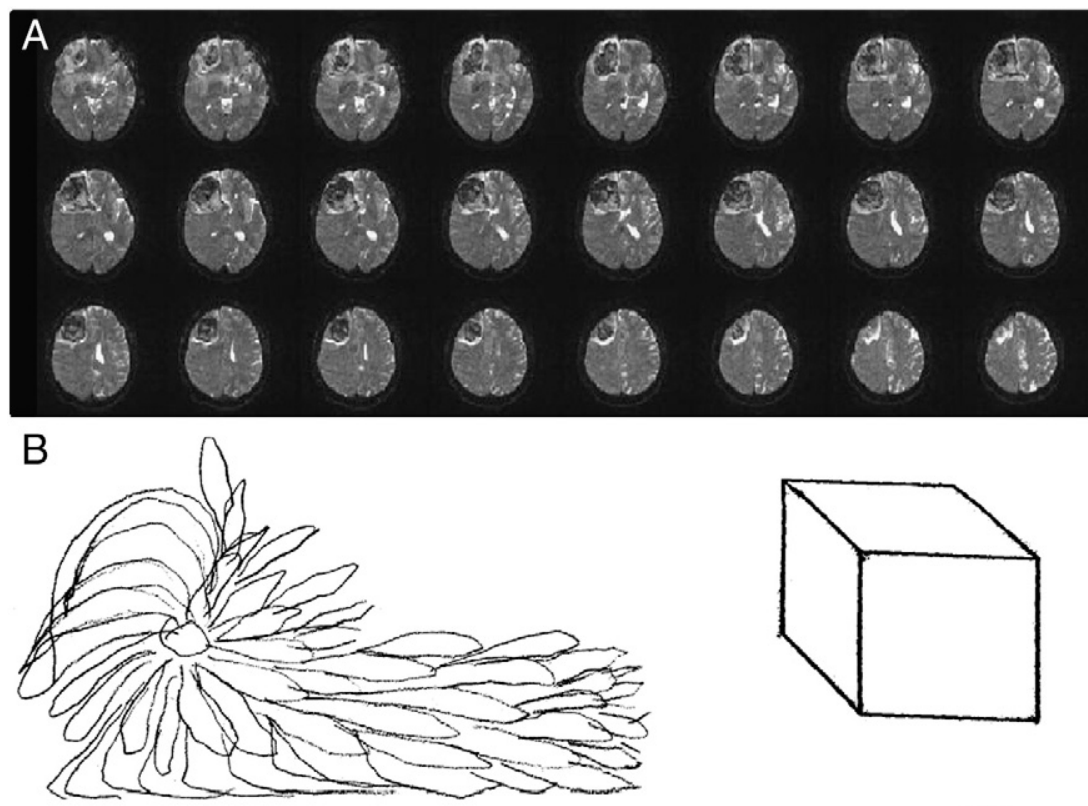

D
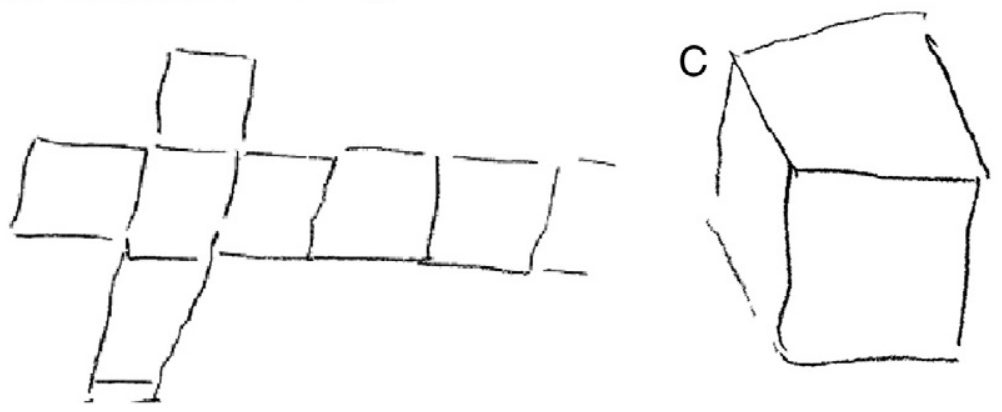

Fig. 1. A) Right hemispheric lesion (MRI axial); B) Draw a flower of memory; C) Copy the drawing of a cube; D) Draw a cube unfolded.

interpreted as a primarily spatial disorder rather than a purely visual one. Indeed, the abnormal right-sided drawings of our patient suggest an object-based disorder [6], but paradoxically ipsilaterally to the lesion. Unlike in other reports, the phenomenon in our patient cannot be explained by perseveration, since motor and particularly graphic perseverations [7] and stimulus-bound behavior [8] were totally absent. We hypothesized that our right side distortion could be due to an ipsilateral relaxation of the spatial medium reflecting a compensatory attentional shift towards the ipsilesional side [1,3], that fit with right hemisphere dominance in the attention network [9].

\section{Conclusion}

The occurrence of such a pure ipsilateral hyperschematia associated with a right frontal lesion confirms that this disorder is independent from spatial neglect and from classic frontal perseverations. Rather, our results support the presence of a spatial component to the perception of object size. This new subtype of hyperschematia might be a specific sub-component of space distortion.

\section{References}

[1] Vallar G, Rode G. Commentary on Bonnier P. L'aschématie. Rev Neurol (Paris) 1905;13:605-9. Epilepsy Behav 2009;16:397-400.

[2] Blom JD. A Dictionary of Hallucinations. New York: Springer; 2010. p. 1-57.

[3] Rode G, Michel C, Rossetti Y, Boisson D, Vallar G. Left size distortion (hyperschematia) after right brain damage. Neurology 2006;67:1801-8.

[4] Rode G, Revol P, Rossetti Y, Vallar G. 3D left hyperschematia after right brain damage. Neurocase 2008;14:369-77.

[5] Vallar G, Papagno C. Pierre Bonnier's (1905) cases of bodily “aschematie”. In: Code C, Wallesch C-W, Joanette Y, Lecours AR, editors. Classic Cases in Neuropsychology 2. Hove, East Sussex: Psychology Press; 2003. p. 147-70.

[6] Milner AD, Harvey M. Distortion of size perception in visuospatial neglect. Curr Biol 1995;5:85-9.

[7] Na DL, Adair JC, Kang Y, Chung CS, Lee KH, Heilman KM. Motor perseverative behavior on a line cancellation task. Neurology 1999;52:1569-76.

[8] Evyapan D, Kumral E. Visuospatial stimulus-bound automatic writing behavior: a right hemispheric stroke syndrome. Neurology 2001;56:245-7.

[9] Corbetta M, Shulman GL. Control of goal-directed and stimulus-driven attention in the brain. Nat Rev Neurosci 2002;3:201-15. 